\title{
A assistência de enfermagem no pré-natal em gestantes diagnosticadas com sífilis: através de um revisão integrativa
}

\author{
Nursing care in prenatal care in pregnant women diagnostic with syphilis: through an \\ integrative review
}

Atención prenatal de enfermería en mujeres embarazadas diagnóstico con sífilis: através de una revisión integrativa

Maria Auxiliadora da Silva ${ }^{1 *}$, Patrícia dos Santos Dantas, Jabneela Vieira Pereira Vetorazo.

\section{RESUMO}

Objetivo: Este estudo delineou descrever as estratégias adotadas por enfermeiros no manejo das gestantes com diagnóstico de sífilis. Métodos: Trata-se de uma revisão integrativa, qualitativa, realizada entre os meses de janeiro de 2020 a março 2021, nas bases de dados da Literatura Latino-Americana e do Caribe em Ciências da Saúde (LILACS), Literatura Internacional em Ciências da Saúde (PUBMED), na Biblioteca Eletrônica Científica Online (Scielo) e Biblioteca Virtual em Saúde (BVS), a partir da inclusão de artigos científicos disponíveis na íntegra, em livre acesso, publicados no período entre 2017 a 2020. Resultados: Foram encontrados 120 artigos nas bases de dados, excluídos 92 após a aplicação dos critérios de exclusão, selecionados 28 artigos para serem lidos na íntegra e, ao final, 21 estudos foram incluídos para serem analisados a fim de subsidiar a revisão de literatura. Considerações finais: Verificou-se que a literatura aponta a importância do profissional da enfermagem em promover ações integrais, que qualifiquem esse atendimento, com bom acolhimento, orientações sobre a necessidade de rastreamento da sífilis durante o Pré-Natal, notificando e realizando busca ativa, a fim de desenvolver um acompanhamento a terapia indicada, e monitorar análises sorológicas evitando complicações e garantindo maior segurança para mãe e para o bebê.

Palavras-chave: Gestantes, Sífilis, Atuação do enfermeiro, Atenção básica.

\begin{abstract}
Objective: This study to describe the strategies adopted by nurses in the management of pregnant women diagnosed with syphilis. Methods: This is an integrative review, qualitative, carried out between of January 2020-March 2021, in the databases of Latin American and Caribbean Literature in Health Sciences (LILACS), International Literature in Sciences da Saúde (PUBMED), in the Online Electronic Scientific Library (Scielo) and Virtual Health Library (VHL), based on the inclusion of scientific articles available in full, in free access, published in the period between 2017 and 2020. Results: 120 articles were found in the databases, 92 were excluded after applying the exclusion criteria, 28 articles were selected to be read in full and, at the end, 21 studies were included to be analyzed in order to support the literature review. Final considerations: It the literature points out the importance of the nursing professional in promoting comprehensive actions that qualify this service, with a good reception, guidance on the need for screening for syphilis still in the Prenatal phase, always notifying, carrying the active search, in order to develop a follow-up to the elaborated therapy, and, to monitor serological analyzes, avoiding complications and ensuring greater safety for the mother and the baby.
\end{abstract}

Key words: Pregnant women, Syphilis, Nurse's work, Primary care.

\footnotetext{
${ }^{1}$ Centro Universitário Aparício Carvalho (FIMCA), Porto Velho - RO. *E-mail: patti.dantas@gmail.com
} 


\section{RESUMEN}

Objetivo: Estudio diseñado para describir las estrategias adoptadas por enfermeras en manejo de gestantes diagnosticadas con sífilis. Métodos: Se trata de una revisión integradora, cualitativa, realizada de enero de 2020 a marzo de 2021, en las bases de datos de Literatura Latinoamericana y Caribeña en Ciencias de la Salud (LILACS), Literatura Internacional en Ciencias de la Salud (PUBMED), en el Biblioteca Científica en Línea (Scielo) y Biblioteca Virtual en Salud (BVS), a partir de la inclusión de artículos científicos disponibles en su totalidad, en libre acceso, publicados en el período comprendido entre 2017 y 2020 . Resultados: Se encontraron un total de 120 artículos en las bases de datos, se excluyeron 92 después de aplicar los criterios de exclusión, se seleccionaron 28 artículos para ser leídos en su totalidad y, al final, se incluyeron 21 estudios para ser analizados. Consideraciones finales: La literatura destaca la importancia del profesional de enfermería en promover acciones integrales, que habiliten este servicio, con buena acogida, orientación sobre la necesidad de cribado de sifilis, notificando y realizando una búsqueda activa para monitorear la terapia y monitorear los análisis serológicos, evitando complicaciones y garantizando una mayor seguridad para la madre y el bebé.

Palabras clave: Mujeres embarazadas, Sífilis, Desempeño de enfermería, Atención primaria.

\section{INTRODUÇÃO}

A Sífilis é uma doença grave e infectocontagiosa sistêmica conhecida como "Cranco Duro", causada pela Bactéria Treponema Pallidum, dividida em primária, secundária e terciária. A transmissão ocorre principalmente por vias sexual ou por via placentária. No Brasil, é registrado um alto nível de infecções principalmente entre as gestantes, causando um alerta para atenção que é prestada e a seriedade na realização e incentivo do pré-natal, no qual pode ser realizado o diagnóstico precoce e tratamento entre essas mulheres, envolvendo a participação do parceiro nesse momento (BRASIL, 2020).

Quando a transmissão é por via placentária durante a gestação, ela é chamada de sífilis congênita. Ela pode ser muito grave, sobretudo quando não tratada, haja vista que, pode evoluir para um quadro crônico, alternando períodos nos quais pode se apresentar mais branda ou aguda podendo afetar a pele, os ossos, podendo até acometer o sistema nervoso central e os órgãos internos (BARROS SMO, 2009).

Embora a sífilis na gestação seja de fácil diagnóstico e de tratamento eficaz, ainda apresenta prevalência alta, estimando que ocorram anualmente 12 milhões de casos na população adulta, desses $90 \%$ em países desenvolvidos, e a sífilis congênita causadora de 500 mil óbitos fetais no mundo. No Brasil, foram registrados entre junho de 2005 a junho de 2019 no Sistema de Agravos de Notificações (SINAN), um total de 324.321 novos casos de sífilis em gestantes notificados, a região Sudeste é a campeã de notificações com (45\%) e a Centro-oeste obtém o menor número com um total de apenas 8,9\% dos registros (BRASIL, 2020).

Versa-se nesse estudo sobre a seriedade do diagnóstico precoce da sífilis em mulheres grávidas, ressaltando que a não realização do diagnóstico no pré-natal, é um dos principais fatores da sífilis congênita. Dentro desse contexto, dar ênfase ao relevante papel do profissional da enfermagem em sensibilizar as pacientes gestantes durante o pré-natal, sobre a necessidade de aderirem ao tratamento completo, tanto ela como seu parceiro, bem como tem-se como objetivo geral, descrever as estratégias adotadas por enfermeiros no manejo das gestantes com diagnóstico de sífilis (SANTOS FP, et al., 2017).

O enfermeiro tem papel essencial na assistência pré-natal, pois possui conhecimento para atuar no mesmo, verificando a para necessidades específicas de cada gestante, com estratégias de assistência para prevenção de doenças, conforme regulamenta a Lei oㅡ 7.498, de 25 de junho de 1986, que dispõe como uma das funções do enfermeiro, a prestação de assistência de enfermagem à gestante, parturiente, puérpera e ao recém-nascido em Unidade de Saúde da Família (USF) como abordado no art.8 em seu inciso II (GOMES CBA, et al., 2017a).

É preocupante que alguns estudos demonstrarem que vem existindo muitas falhas no diagnóstico da sífilis congênita durante o pré-natal, muitas gestantes nem chegam a realizar o exame (FIGUEREDO-FILHO, et al., 
2012). Logo, questiona-se nesse estudo, o que apresenta a literatura pertinente, sobre os fatores assistenciais da enfermagem que têm contribuído para a ocorrência dessas falhas.

Diante ao exposto, o presente estudo tem como objetivo de aprofundar o entendimento sobre a sífilis congênita, abordar como ocorre a transmissão, sobre a assistência da enfermagem em relação ao diagnóstico durante o pré-natal, como é realizado os exames, que tipo de capacitação o profissional da enfermagem recebe para realizá-lo, tudo isso por meio de uma revisão bibliográfica, com publicações de anos recentes.

\section{MÉTODOS}

Trata-se de uma revisão integrativa da literatura, utilizada por seu potencial de organização, síntese de conhecimentos e identificação de lacunas que contribuem na análise crítica de um objeto de estudo proposto. Para isso, seguiu-se os seguintes passos para seu desenvolvimento: formulação da questão norteadora; busca na literatura; extração dos dados dos estudos selecionados; avaliação dos estudos; interpretação e síntese dos resultados; apresentação da revisão integrativa.

Para responder ao objetivo do presente estudo, formulou-se a seguinte questão norteadora: "Quais estratégias vêm sendo adotadas por enfermeiros no manejo da sífilis em gestantes?"

O levantamento bibliográfico foi realizado nas bases de dados da Literatura Latino-Americana e do Caribe em Ciências da Saúde (LILACS), Literatura Internacional em Ciências da Saúde (PUBMED), na Biblioteca Eletrônica Científica Online (Scielo) e Biblioteca Virtual em Saúde (BVS), o que possibilitou a busca por estudos publicados no cenário nacional.

Para a seleção dos estudos, optou-se pela inclusão de artigos científicos disponíveis na íntegra, em livre acesso, publicados no período entre 2017 a 2020, nos idiomas português, bem como aqueles que, após leitura do título e resumo, abordassem aspectos relacionados à temática do estudo. Foram excluídos os artigos duplicados, artigos de revisão, de reflexão/debates, monografias, dissertações, teses, comentários, editoriais e cartas.

Os estudos elegíveis foram lidos na íntegra, a fim de incluir ou não, aqueles que conseguissem responder à questão norteadora. A interpretação e síntese dos resultados encontrados será apresentada em uma tabela contendo os autores, ano, local e periódico de publicação, bem como os principais resultados quanto a assistência de enfermagem no manejo da sífilis em gestantes.

\section{RESULTADOS}

Foram encontrados 124 artigos nas bases de dados, dos quais em um primeiro momento, 82 artigos foram excluídos após aplicação dos critérios: foram excluídos 10 estudos por estarem indisponíveis, 12 por estarem inferiores ao período previamente selecionado, 19 por estarem duplicados, 13 por serem artigos de revisão, 14 por serem de outro idioma, 11 por serem teses e dissertações, 13 por apresentarem apenas título e resumo. Após a leitura de título e resumo, 32 artigos foram selecionados para leitura na íntegra, e após serem lidos na íntegra, 11 artigos foram excluídos, o que possibilitou ao final, a inclusão de 21 artigos para serem analisados a fim de subsidiar a revisão de literatura (Figura 1). 
Figura 1 - Fluxograma das etapas para a seleção dos artigos desta revisão integrativa.

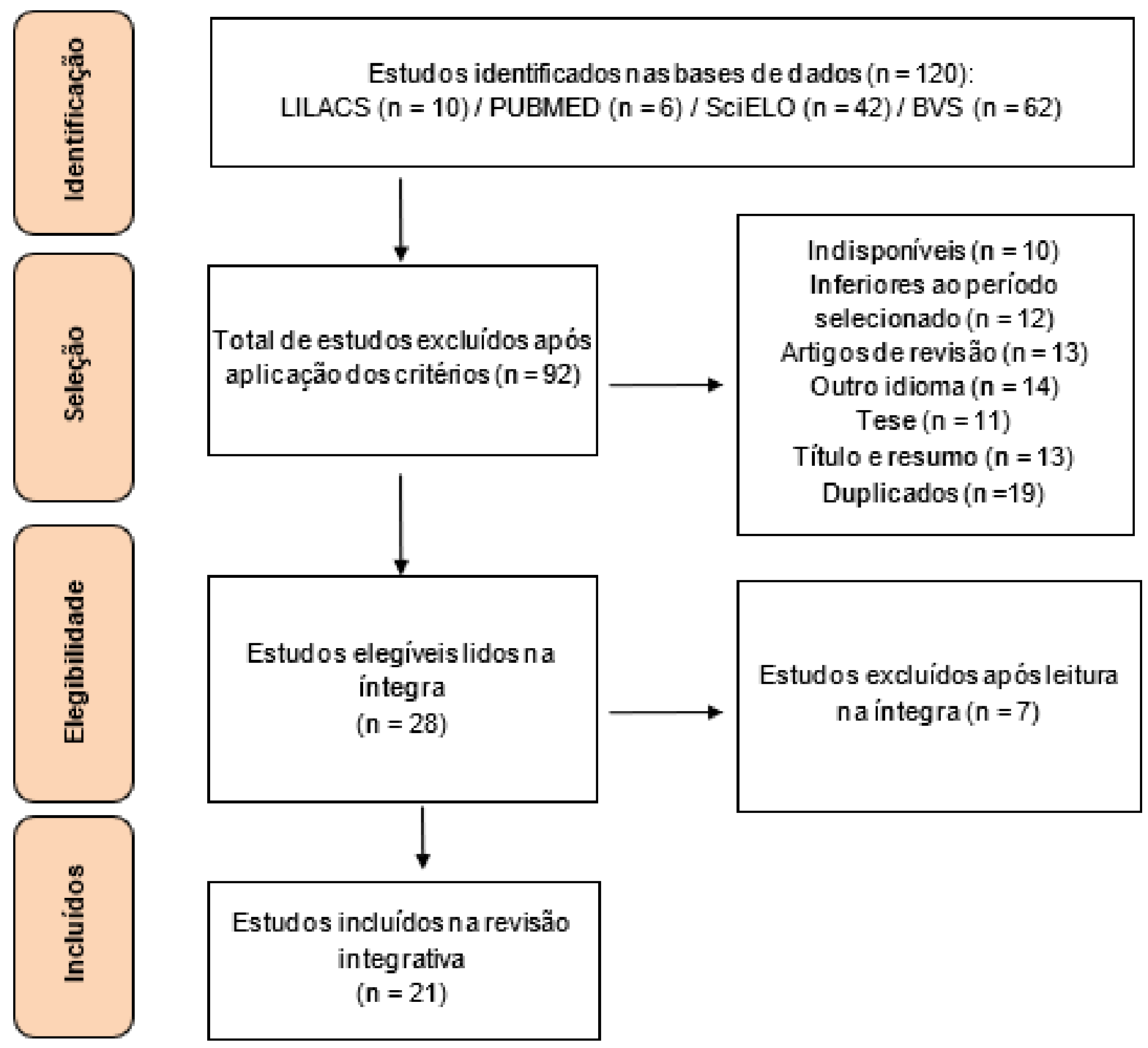

Fonte: Silva MA, et al., 2021.

Dos artigos incluídos na revisão, a maioria foi publicado no idioma português (99\%), e apenas um no idioma inglês (1\%), que foi traduzido. Quanto ao tipo de estudo, identificou-se majoritariamente, pesquisas do tipo observacionais (28\%), Experimental $(5 \%)$, transversais analíticos $(14 \%)$, transversais com abordagens qualitativas (29\%), quantitativa (24\%) (Quadro 1). 


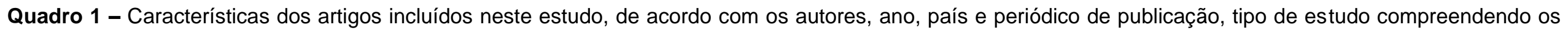
fatores atribuídos assistência de enfermagem no manejo da sífilis em gestantes.

\begin{tabular}{|c|c|c|c|}
\hline Autores & $\begin{array}{l}\text { Ano, cidade e periódico de } \\
\text { publicação }\end{array}$ & $\begin{array}{l}\text { Tipo de } \\
\text { estudo }\end{array}$ & Vertente do artigo \\
\hline SANTANA MVS, et al. & $\begin{array}{l}2019 \text { / Maceió }(A L) \text { / Diversitas } \\
\text { Journal }\end{array}$ & Observacional & Sífilis gestacional na atenção básica. \\
\hline CÉSAR JA, et al. & $\begin{array}{l}2020 \text { / Rio de Janeiro (RJ) / } \\
\text { Revista Bras Epidemiologia. }\end{array}$ & $\begin{array}{l}\text { Transversal } \\
\text { qualitativo }\end{array}$ & $\begin{array}{l}\text { Não realização de teste sorológico para sífilis durante o pré-natal: prevalência e } \\
\text { fatores associados. }\end{array}$ \\
\hline SANTANA FP, et al. & $\begin{array}{l}\text { 2017/ Itabaiana (SE)/ Rev. bras. } \\
\text { Enferm }\end{array}$ & Observacional & $\begin{array}{l}\text { Prevenção da Sífilis Congênita e as Intervenções do Enfermeiro na Saúde } \\
\text { Pública. }\end{array}$ \\
\hline SANTOS FP, et al. & $\begin{array}{l}2017 \text { / Belo Horizonte (MG)/ } \\
\text { Rev. bras. Enferm }\end{array}$ & $\begin{array}{l}\text { Transversal } \\
\text { qualitativo }\end{array}$ & Sífilis na gestação: A importância do diagnóstico precoce. \\
\hline SILVA JGD, et al. & $\begin{array}{l}2019 \text { / Porto Alegre (RS) / Rev. } \\
\text { Cogitare enferm. }\end{array}$ & $\begin{array}{l}\text { Transversal } \\
\text { analítico }\end{array}$ & Sífilis gestacional: Repercussões para a puérpera. \\
\hline ROSA RFN, et al. & $\begin{array}{l}2020 \text { / Paulo Afonso (BA) / Rev. } \\
\text { enferm UFPE online }\end{array}$ & $\begin{array}{l}\text { Transversal } \\
\text { qualitativo }\end{array}$ & O manejo da sífilis gestacional no pré-natal. \\
\hline CONCEIÇÃO HN, et al. & $\begin{array}{l}2019 \text { / Caxias (MA) / Saúde \& } \\
\text { debate. }\end{array}$ & $\begin{array}{l}\text { Transversal } \\
\text { qualitativo }\end{array}$ & Análise epidemiológica e espacial dos casos de sífilis gestacional e congênita. \\
\hline GUIMARÃES TA, et al. & $\begin{array}{l}2018 \text { / São Luís (MA) / Revista } \\
\text { Arq. Cienc. Saúde }\end{array}$ & Observacional & Sífilis em gestantes e sífilis congênita no Maranhão. \\
\hline NOGUCHI TFB, et al. & $\begin{array}{l}2018 \text { / Vale do Rio Verde (MG) / } \\
\text { Revista da Universidade Vale do } \\
\text { Rio Verde }\end{array}$ & $\begin{array}{l}\text { Transversal } \\
\text { qualitativo }\end{array}$ & $\begin{array}{l}\text { Prevalência de sífilis em gestante em uma cidade do sul de Minas Gerais: análise } \\
\text { documental. }\end{array}$ \\
\hline FILHO SS, et al. & $\begin{array}{l}2018 \text { / Teixeira de Freitas (BA) / } \\
\text { Revista Mosaicum }\end{array}$ & $\begin{array}{l}\text { Transversal } \\
\text { analítico }\end{array}$ & $\begin{array}{l}\text { Incidência de sífilis em gestantes atendidas nas unidades de saúde no } \\
\text { município de Teixeira de Freitas, Bahia. }\end{array}$ \\
\hline FELIPE LAF, et al. & $\begin{array}{l}2020 \text { / Rio de Janeiro (RJ) / } \\
\text { Enferm Bras. }\end{array}$ & $\begin{array}{l}\text { Transversal } \\
\text { quantitativo }\end{array}$ & $\begin{array}{l}\text { O perfil epidemiológico das gestantes notificadas com sífilis no município do Rio } \\
\text { de Janeiro - } 2008 \text { a } 2017 \text {. }\end{array}$ \\
\hline
\end{tabular}




\begin{tabular}{|c|c|c|c|}
\hline Autores & $\begin{array}{l}\text { Ano, cidade e periódico de } \\
\text { publicação }\end{array}$ & $\begin{array}{l}\text { Tipo de } \\
\text { estudo }\end{array}$ & Vertente do artigo \\
\hline SOUSA FDC, et al. & $\begin{array}{l}2020 \text { / Araraquara (SP) / Revista } \\
\text { Brasileira Multidisciplinar }\end{array}$ & $\begin{array}{l}\text { Transversal } \\
\text { quantitativo }\end{array}$ & Perfil da Sífilis na Gestação no Período de 2007/2016 em Caxias-MA. \\
\hline LINDOSO RF, et al., & $\begin{array}{l}2020 \text { / São Luiz (MA) / Braz. J. } \\
\text { Hea. Rev. }\end{array}$ & $\begin{array}{l}\text { Transversal } \\
\text { qualitativo }\end{array}$ & $\begin{array}{l}\text { Aspectos epidemiológicos dos casos de gestantes com sífilis em São Luís-MA no } \\
\text { período de } 2010 \text { a } 2015 \text {. }\end{array}$ \\
\hline GOMES CBA, et al. & $\begin{array}{l}\text { 2017a / Uberlândia (MG) / } \\
\text { Scientia Plena }\end{array}$ & $\begin{array}{l}\text { Transversal } \\
\text { Analítico }\end{array}$ & $\begin{array}{l}\text { Perfil epidemiológico dos casos de sífilis congênita no estado de Minas Gerais } \\
\text { no período de } 2007 \text { a } 2017 \text {. }\end{array}$ \\
\hline LIMA LE, et al. & $\begin{array}{l}2019 \text { / São Paulo (SP) / J Health } \\
\text { Sci Inst. }\end{array}$ & $\begin{array}{l}\text { Transversal } \\
\text { quantitativo }\end{array}$ & $\begin{array}{l}\text { Conhecimento das gestantes com sífilis sobre a doença e perfil sociodemográfico } \\
\text { em uma UBS e Hospital Maternidade da zona norte de São Paulo. }\end{array}$ \\
\hline GONÇALVES MM, et al. & $\begin{array}{l}2020 \text { / Fortaleza (CE) / Rev. } \\
\text { Mult. Psic. }\end{array}$ & $\begin{array}{l}\text { Transversal } \\
\text { quantitativo }\end{array}$ & Os Desafios no Tratamento da Sífilis Gestacional. \\
\hline GOMES CBA, et al. & $\begin{array}{l}\text { 2017b / Florianópolis (SC) / } \\
\text { Texto \& Contexto Enfermagem. }\end{array}$ & Observacional & Consulta de Enfermagem no Pré-natal: Narrativas de gestantes e enfermeiras. \\
\hline NUNES JT, et al. & $\begin{array}{l}2017 \text { / São Paulo (SP) / Ref. } \\
\text { enferm UFPE online }\end{array}$ & $\begin{array}{l}\text { Transversal } \\
\text { quantitativo }\end{array}$ & Sífilis Na Gestação: Perspectivas e Condutas do Enfermeiro. \\
\hline OLIVEIRA JAC; , et al. & $\begin{array}{l}2017 \text { / Fortaleza (CE) / Revista } \\
\text { Científica Interdisciplinar. }\end{array}$ & Observacional & Assistência de enfermagem no pré-natal em relação à sífilis congênita. \\
\hline SOARES LG, et al. & $\begin{array}{l}2017 \text { / Recife (PE) / Rev. Bras. } \\
\text { Saúde Matern. Infant. }\end{array}$ & Experimental & $\begin{array}{l}\text { A Sífilis gestacional e congênita: características maternas, neonatais e desfecho } \\
\text { dos casos. }\end{array}$ \\
\hline FAVERO ML, et al. & $\begin{array}{c}2019 \text { / Fortaleza (CE) / J. Health } \\
\text { Biol Sci }\end{array}$ & $\begin{array}{c}\text { Transversal } \\
\text { qualitativo }\end{array}$ & Sífilis congênita e gestacional: notificação e assistência pré-natal. \\
\hline
\end{tabular}

Fonte: Silva MA, et al., 2021. 


\section{DISCUSSÃO}

A literatura consultada trás subsídios para que se possa compreender os fatores atribuídos assistência de enfermagem no manejo da sífilis em gestantes.

Evidenciou-se nesse estudo que, a Atenção Básica de Saúde (UBS) é a principal porta de entrada dos serviços em saúde, oferece um trabalho voltado à saúde da mulher e ao pré-natal. A consulta pré-natal foi implantada em 1994, os profissionais de saúde envolvidos médico ou enfermeiro, devem compreender o significado da gestação para a mulher, criar uma situação de amizade e de confiança com a gestante (NUNES JT, et al., 2017; GOMES CBA, et al., 2017a).

A assistência de enfermagem volta-se ao contato direto com os pacientes, orientando, diagnosticando, identificação dos sinais e sintomas da doença, bem como acompanhar e direcionar tratamento com foco no binômio mãe-bebê e sua rede familiar diante da Sífilis Gestacional e Congênita (SILVA JG DA, et al., 2019).

$\mathrm{Na}$ Atenção básica, durante o pré-natal, o profissional enfermeiro(a) inicia o pré-natal, e na primeira consulta solicita os exames de rotina, além de cadastrar a gestante e classificá-la em um grupo, que pode ser de baixo ou alto risco (PINTO JC, et al., 2020). Ressalta-se que a responsabilidade do enfermeiro (a) é essencial no combate da sífilis gestacional, pois tem como escopo fazer todo acompanhamento da gravidez, minimizando os riscos tanto para mãe como para o bebê, atuando na prevenção da sífilis congênita e detecção precoce da sífilis materna, (LIMA LE, 2019, LINDOSO RF, et al., 2020).

O manejo ocorre da seguinte forma, no decorrer do pré-natal o enfermeiro (a) realiza o teste rápido com o intuito de diagnosticar sífilis na primeira consulta; posteriormente, no terceiro trimestre da gestação, no parto e se necessário no puerpério, o teste é repetido. Ressaltando que, essa gestante deve aderir ao pré-natal com mínimo de 6 consultas, compreendendo o terceiro trimestre. Logo o enfermeiro deve incentivar os agentes de saúde, a captar as gestantes para não iniciar o pré-natal tardiamente e para dar continuidade, de forma a reduzir o número de casos de sífilis materna, e ainda para que se inicie o tratamento precoce, adotando medidas de prevenção para evitar o complicações da infecção (LINDOSO RF, et al., 2020, GONÇALVES MM, et al., 2020).

Os profissionais enfermeiros atuam com base em protocolos do Ministério da saúde, os quais conferem mais autonomia na abordagem da doença (NOGUCHI TFB, et al., 2018). O profissional de enfermagem atua por meio de educação em saúde com ênfase no direcionamento, localização de situações de risco e na educação para a saúde. Fazendo-se possível evitar, desta maneira, a transmissão vertical e progresso da sífilis congênita e de outras doenças infecciosas e contagiosas (OLIVEIRA DR, et al., 2017).

Outra função da enfermagem, é realizar a notificação de novos casos da doença ao SINAN, pois tais dados subsidiarão ações de controle da doença (SANTANA EP, et al., 2019). A notificação trata-se de uma ferramenta imprescindível para a vigilância epidemiológica, entretanto, como vem sendo descrito na literatura consultada, a subnoticação ainda é um desafio, tendo em vista que os números apontados não são fidedignos (SOARES LG, et al.,2017; SANTOS FP, et al., 2017).

Também cabe ao enfermeiro (a) promover campanhas educativas, falando sobre a sífilis, a prevenção, o tratamento e profilaxia sobre o ressurgimento dessa doença, sobre a importância da realização do teste rápido tanto para a gestante quanto para o recém-nascido e para a população, contribuindo assim, para a diminuição dos índices da doença e suas complicações (SANTANA MVS, 2019; CÉSAR JA, et al., 2020; SANTANA EP, et al., 2017).

Outro ponto relativo à assistência da enfermagem está relacionado ao impacto do diagnóstico de sífilis, sendo relatado pelas gestantes sentimentos como: ansiedade, medo e alteração na autoestima que ocorrem por conta da falta de conhecimento referente a doença e das possíveis complicações que possam ocorrer advindas da mesma (SANTOS FP, et al., 2017).

Nesse sentido, o profissional da enfermagem precisa estar apto a criar vínculos com pacientes, mantendo contato direto com as gestantes, a fim de que possam realizar os testes rápidos e identifique os sintomas da sífilis ainda no início do pré-natal, sempre orientando a gestante, parceiro e toda família, buscando garantir a integralidade do cuidado desde a detecção, diagnóstico até o tratamento da sífilis (SILVA JG DA, et al., 2019; SANTOS FP, et al., 2017). 
Estudos de César JA, et al. (2020), apontam para o fato de que apesar de estar comprovado que o diagnóstico em momento adequado da sífilis na gravidez previna complicações, detecta-se falhas no manejo da assistência da enfermagem, ao se observar o predomínio do diagnóstico tardio, erros no manejo do tratamento, visto que a totalidade dos tratamentos foi considerada inadequada, segundo o Ministério da Saúde. Outros dados que demonstram a fragilidade do serviço de assistência da enfermagem são: a não adesão ao tratamento pela gestante e do parceiro, poucas consultas pré-natais, enfermeiros que não sentem-se seguros em realizar os esquemas de tratamento, falta de orientações sobre a doença e sobre uso de preservativos, quebra da continuidade do cuidado com mudança de unidade de saúde durante a assistência, e problemas organizacionais dos serviços de saúde (GUIMARÃES TA, et al., 2018, FAVERO LDC, et al., 2019, FELIPE LAF, et al., 2020, SANTOS FP, et al., 2017, ROSA RFN, et al., 2020).

A literatura consultada apontou o predomínio da sífilis congênita em crianças filhas de mães que realizaram o acompanhamento pré-natal e o diagnóstico da infecção materna, durante o parto ou a curetagem, de forma a entender que ocorreu pré-natal tardio, sendo assim, detectada falha na assistência da enfermagem para 0 manejo correto da infecção (GUIMARÃES TA, et al., 2018, FAVERO LDC, et al., 2019, FELIPE LAF, et al., 2020, SANTOS FP, et al., 2017, ROSA RFN, et al., 2020).

Face ao exposto, conclui-se que a qualidade da atenção pré-natal deve ser reorganizada, sendo este o principal desafio para o controle da sífilis gestacional e consequentemente da sífilis congênita, fato que ainda não vem ocorrendo de forma efetiva em todo Brasil, enfatizando que é necessário que universalize a oferta de cuidados e elimine as oportunidades perdidas de assistência à gestante (SIEPIERSKI FILHO S, et al., 2018).

Os índices deste agravo ainda se apresentam altos, de forma se considerar que as ações de diagnóstico e prevenção realizadas pela assistência de enfermagem precisam ser reforçadas, não somente no pré-natal e parto, como ainda antes da gestação ocorrer, além de se enfatizar as informações sobre a doença (SOUZA FDC, et al,. 2020).

A falta de informação é citada na literatura analisada, bem como a assistência planejar e realizar ações conscientizadoras e preventivas sobre a importância da prevenção durante a relação sexual, do uso dos contraceptivos, como também a importância do teste (GONÇALVES MM, et al., 2020; SOUZA FDC, et al,. 2020; SOARES LG, et al., 2017).

Diante de tantas falhas e fragilidades na assistência, considera-se ser necessário que, os profissionais que atuam diretamente com as gestantes recebam capacitações e treinamentos para melhor assistir as pacientes com sífilis e para prevenir a sífilis congênita (LIMA LE, et al., 2019; FAVERO LDC, et al., 2019, SANTANA EP, et al., 2017). Ressalta-se a necessidade de estratégias inovadoras como, entrevistas e visitas domiciliares que são indispensáveis para a conscientização do problema para a gestante e para o feto, visto que para se alcançar sucesso na terapia do referido agravo, depende-se diretamente do controle exercido por um Serviço de Enfermagem de Saúde Pública (SANTANA EP, et al., 2017; CONCEIÇÃO HN, et al., 2019; GUIMARÃES TA, et al., 2018; ROSA RFN, et al., 2020).

Salienta-se que, o profissional de enfermagem deve incentivar a participação do parceiro no decorrer do pré-natal, para que receba as mesmas orientações que a mãe irá receber, a fim de se conseguir um tratamento eficiente e conseguindo prevenir a sífilis congênita (MESQUITA KO, et al., 2012). Portanto, para que haja o manejo adequado da sífilis congênita, deve-se zelar por uma assistência capacitada para identificar precocemente a gestante infectada e esteja apta a acompanhar e tratar adequadamente a paciente. A equipe deve estar integrada e comprometida em oferecer um excelente pré-natal, visto que este é um direito de toda gestante e dever do profissional de saúde em desempenhá-lo adequadamente (SANTOS FP, et al., 2017).

\section{CONSIDERAÇÕES FINAIS}

Verificou-se nesse estudo que a assistência de enfermagem é fundamental para redução do índice de sífilis congênita, devido a possibilidade de criação de vínculo com a comunidade, em especial as mulheres gestantes, de forma que atuam como maior veículos de informação na atenção primária, e precisam para isso 
ter o ciência do manejo desta doença, de forma a levar a um desfecho favorável, cooperando com a criação de estratégias que marquem caminhos para uma assistência qualificada. Todavia, vários estudos apontaram falhas nessa assistência, concordando que sejam introduzidas políticas públicas que promovam treinamentos aos enfermeiros para que desenvolvam uma assistência voltada para gestantes e seus respectivos parceiros. Enfatiza-se a importância de o profissional da enfermagem promover ações integrais, que qualifiquem esse atendimento, com um bom acolhimento, orientações sobre a necessidade de rastreamento da sífilis ainda na fase do Pré-Natal, a fim de ter um controle dos casos na equipe, notificando sempre, realizando busca ativa, a fim de desenvolver um acompanhamento a terapia indicada, e, para tanto monitorar análises sorológicas evitando complicações e garantindo maior segurança para mãe e para o bebê.

\section{REFERÊNCIAS}

1. BARROS SMO. Enfermagem obstétrica e ginecológica: guia para prática assistencial. 2.ed. São Paulo: Roca, 2009; $488 \mathrm{p}$.

2. BRASIL. Manual do Ministério da Saúde. Guia do Pré-Natal do Parceiro para Profissionais de Saúde. Rio de Janeiro, RJ: Ministério da Saúde, 2016.

3. CESAR JA, et al. Não realização de teste sorológico para sífilis durante o pré-natal: prevalência e fatores associados. Rev. Bras. Epidemiologia. Rio de Janeiro, 2020; 23(2): 1980-5497.

4. CONCEIÇÃO HN, et al. Análise epidemiológica e espacial dos casos de sífilis gestacional e congênita. Saúde Debate, 2019; 43(123):2358-2898.

5. FAVERO MLDC, et al. Sífilis congênita e gestacional: notificação e assistência pré-natal. Archives. Health Sciences. 2019; 26(1): 2318-3691.

6. FELIPE LAF, et al. O perfil epidemiológico das gestantes notificadas com sífilis no município do Rio de Janeiro - 2008 a 2017. Enfermagem Brasil, 2020; 19(1).

7. FILHO EAF, et al. Sífilis e Gestação: Estudo Comparativo de dois períodos (2006 e 2011) em população de puérperas. DST- Jornal Brasileiro de Doenças Sexualmente Transmissível, 2012; 24(1): 2177-8264.

8. GOMES FT, et al. Perfil epidemiológico dos casos de sífilis congênita no estado de Minas Gerais no período de 2007 a 2017a. Scientia Plena, 2020; 16(3): 03741.

9. GOMES CBA, et al. Consulta De Enfermagem No Pré-natal: Narrativas De Gestantes E Enfermeiras. Texto \& Contexto Enfermagem. Florianópolis, 2017b; 28(44):1980-265X.

10. GONÇALVES MM, et al. Os Desafios no Tratamento da Sífilis Gestacional. Revista Multidisciplinar e de Psicologia, 2020; 14(49): 2323-9187.

11. GUIMARÃES TA, et al. Sífilis em gestantes e sífilis congênita no Maranhão. Arquivos de Ciências da Saúde, 2018; 25(2): 2318-3691.

12. LIMA LE, et al. Conhecimento das gestantes com sífilis sobre a doença e perfil sociodemográfico em uma UBS e Hospital Maternidade da zona norte de São Paulo. J Health Sci. Inst., 2019; 37(3): 1140-638.

13. LINDOSO RF, et al. Aspectos epidemiológicos dos casos de gestantes com sífilis em São Luís-MA no período de 2010 a 2015. Brazilian Journal of Health Review, 2020. 3(2): 2595-6825.

14. MESQUITA KO, et al. Análise dos casos de sífilis congênita em Sobral, Ceará: contribuições para assistência prénatal. DST- J Bras. Doenças Sex. Transm., 2012; 24(1): 2177-8264.

15. NOGUCHI TFB, et al. Prevalência de sífilis em gestante em uma cidade do sul de minas gerais: análise documental. Revista da Universidade Vale do Rio Verde, 2018; 16(3): 1517-0276.

16. NUNES JT, et al. Sífilis Na Gestação: Perspectivas E Condutas Do Enfermeiro. Revista de enfermagem UFPE online, 2017; 11(12): 1981-8963.

17. ROSA RFN, et al. O manejo da sífilis gestacional no pré-natal. Revista de enfermagem UFPE online, $2020 ; 14$ : 19818963.

18. SANTANA EP, et al. Prevenção da Sífilis Congênita e as Intervenções do Enfermeiro na Saúde Pública. Revista brasileira de enfermagem, 2017; 4(2): 6087-2186.

19. SANTANA MVS, et al. Sífilis gestacional na Atenção Básica. Diversitas Journal, 2019; 4(2): 2525-5215.

20. SANTOS FP, et al. Sífilis na gestação: a importância do diagnóstico precoce. Revista brasileira de Enfermagem, 2017; 9(12): 6087-2186.

21. SIEPIESKI FILHO S, et al. Incidência de sífilis em gestantes atendidas nas unidades de saúde no município de Teixeira de Freitas, Bahia. Revista Mosaicum, 2018; 27(1): 1980-4180.

22. SILVA JGDA, et al. Sífilis gestacional: repercussões para a puérpera. Cogitare enfermagem, 2019; 24(s/n): 21769133.

23. SOARES LG, et al. Sífilis gestacional e congênita: características maternas, neonatais e desfecho dos casos. Revista Brasileira Saúde Materno e Infantil. Recife, 2017; 17(4): 1806-9304.

24. SOUSA FDSC, et al. Perfil da Sífilis na Gestação no Período de 2007/2016 em Caxias-MA. Revista Enfermagem Atual, 2020; 91(29): 2447-2034. 